\title{
Simulation modelling and resource allocation in complex services
}

\section{Steffen Bayer}

Correspondence to Dr Steffen Bayer, Program in Health Services \& Systems Research, Duke-NUS Graduate Medical School Singapore, 8 College Road, Singapore 169857, Singapore; steffen.bayer@duke-nus.edu.org

Accepted 10 December 2013 Published Online First 17 January 2014

\section{SLinked}

http://dx.doi.org/10.1136/ bmjqs-2013-002107

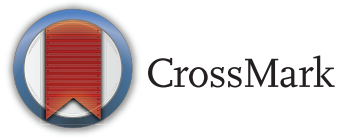

To cite: Bayer S. BMJ Qual Saf 2014;23:353-355.
Allocating resources to healthcare services is difficult and often contested-not least in the context of funding shortfalls and rises in demand. Tako and colleagues ${ }^{1}$ report on the use of simulation modelling to plan capacity investments in a service which is expecting a significant increase in demand. The obesity service in the English hospital they studied required an investment to manage waiting times and meet future anticipated demand. The authors show how simulation modelling can be used to balance investments between different parts of a service and to deal with the uncertainty inherent in planning in a healthcare setting. While their work concerns an obesity service, the need to balance capacities (and therefore investments) will be familiar to many healthcare practitioners across diverse clinical domains. Their simulation shows how investments in capacities in different parts of the service as well as measures to manage demand can be balanced in the specific case of the service they were investigating.

For most readers the conclusions that Tako et al draw for the particular service they studied might be of less relevance than the modelling approach they have taken to arrive at these conclusions. Healthcare managers are frequently required to devise an allocation of resources-to meet rising demand while meeting acceptable performance standards, as in the paper by Tako et al, or to achieve other improvements in efficiency, effectiveness or patient experience. In clinical medicine, new treatments enter practice after trials establish their efficacy and safety. Before introducing new service innovations, one would hope that, at a minimum, a pilot study would examine the consequences of the service innovation.

When allocating resources, we would similarly desire a safe and cost-effective way to examine the possible consequences of the investment in advance. Even experienced managers might struggle to predict the consequences of increasing capacities in different parts of a complex service system comprising multiple resources and pathways. In such situations, having an approach which aids understanding of the relationships between parts of the system and which allows testing different resource allocations and policies in a virtual experiment before committing to specific real investments (and reaping real consequences) constitutes a tremendous boon.

Tako et al describe the obesity service as an interconnection of multiple microsystems consisting of individual clinics, ranging from dietary advice and medical treatment to surgery. Of course, this interconnectedness does not represent a unique characteristic of the service they studied-many healthcare services are of this nature. Indeed, some healthcare services that at first appear unconnected and isolated, on more careful consideration prove to be influenced by other services and in turn influence other services.

As in the case studied in this paper, ${ }^{1}$ feedback between different parts of the care system and bottlenecks along a care pathway can result in one part of the system being affected by investments in another. Their model allows the authors to identify the impacts that changes in patient referral rates and resources (surgeons and physicians) exert on waiting times. For other investments, the relevant resources and outcome variables might differ. However, whether we invest in bed capacity, operating theatre slots or physiotherapy hours, and whether the outcomes we care about are costs, numbers of patients treated or quality indicators, the basic analytical problem remains the same and can benefit from similar simulation modelling approaches to identify solutions.

Tako et al employed discrete event simulation, allowing the authors to model individual patients in their journey through the system and account for the 
effects of random variation (eg, in demand). Discrete event simulation is often the methodology of choice for operational problems involving sequential events in which individuals (customers, patients) consume resources (staff time, beds, slots in clinics or operating theatres, etc.) and where lack of resources might lead to queuing and delays. ${ }^{2}$

Often in such operational problems, the availability of a resource at a particular point in time for a particular patient arriving in some unplanned, random fashion plays an important role-that, on average, a bed would be available holds little meaning for a patient who needs a bed right now. Other problems in healthcare are of a more strategic nature. The consideration of aggregate numbers of patients is often sufficient in such problems. This is the domain of system dynamics modelling. ${ }^{3}$ System dynamics modelling is particularly well suited for problems where delays and feedback between different parts of a system are important and a 'whole system perspective' crucial. Another approach to simulation modelling, less frequently used in healthcare than discrete event simulation and system dynamics, is agent-based modelling. Here the behaviour of individual agents is modelled, with the behaviour of the overall system arising as a consequence of the interacting agents. This approach lends itself in particular to situations where individual choice is crucial (eg, use of discretionary services or behaviours that increase the risk of infections). Other problems will call for techniques such as Markov modelling, structural equation modelling or process mapping.

Tako et al's paper illustrates some of the important challenges often encountered in simulation modelling: access to accurate data is not available. While lack of reliable or comprehensive data can present difficulties for modelling, these data limitations do not necessarily constitute insurmountable problems. Sensitivity analyses can reveal the circumstances under which the model's conclusions remain robust. Decision-making does not necessarily need an exact prediction of, for example, waiting times or bed occupancy rates resulting from an investment; often one requires a reliable assessment indicating which of several options is most promising. Experience frequently shows that such a 'reliable assessment' is less sensitive to precision in data inputs than initially thought. If, however, sensitivity analysis does indeed show that conclusions critically depend on precision in parameters for which no reliable estimates exist, then, at a minimum, this finding highlights where further data collection should focus. This insight into what critical pieces of information are required for robust decision-making is valuable in itself.

Modelling can provide a useful approach to answering specific practical questions, such as what is the best choice among several investment options. Focusing on clearly articulated questions can inform how to deal with the challenge of imprecise data.
A broad focus on 'the system' is rarely productive. Modelling the system is impossible-healthcare is always more complex than a model. Not 'modelling the system' is not only a necessity, but also a virtue: a concise and understandable model which addresses the main features of the system relevant for the question under study and which behaves reliable enough as the real system would is preferable to a comprehensive model which is intractable and incomprehensible.

Of course, this is not a license for the modeller to leave key features out or to commit the error of fitting the data for the wrong reasons. In order to have confidence in a model and its behaviour under a range of scenarios, a model must replicate the mechanism producing the behaviour relevant to answer the question under study - a model that fails to do so is deficient. Producing a simple model without being simplistic represents one of the cornerstones of the art of model building. Simplifications and assumptions in a model have to be judged from a perspective based on the purpose of the modelling exercise, not the comprehensiveness of the model. Tako et al, for example, quite appropriately disregarded operating theatre availability and dieticians' working time, as their focus was on the investment in doctors and surgeons as the scarcest and most expensive resource.

Computer simulation modelling can, as Tako et al's study shows, provide the basis for better investment decisions by supporting experimentation in a safe environment. Such simulation modelling allows examining the consequences of different decisions in a quick, inexpensive and risk free way. Other than informing decisions regarding optimal allocation of resources, simulation modelling has found application to a diverse range of healthcare planning problems. Operational planning of emergency departments, ${ }^{4}$ assessment of screening programmes, ${ }^{5}$ treatment of infectious diseases, ${ }^{6}$ exploration of the impacts of telecare on containing costs and reducing numbers of institutionalised elders, ${ }^{7}$ and predicting the rising burden of informal care provision as a consequence of specific long-term care policies ${ }^{8}$ provide only some of the example applications of simulation modelling to healthcare planning problems. Modelling applications have focused on the patient and provider levels, as well as on individual units and hospital, regional and national levels. Costs, outcomes, demographic change, waiting times and many other issues were analysed with simulation models. Despite the significant history of modelling in diverse application areas in healthcare, the penetration in the healthcare arena has yet to catch up with the widespread use in other industries and fields. ${ }^{9} 10$

Nonetheless, applying modelling in healthcare settings has its challenges. The large number of models with key findings that have not been implemented testifies to the difficulties involved. ${ }^{11-13}$ The complexity of healthcare settings (often with multiple decision- 
makers and stakeholders) makes modelling difficult but also underscores its worth. Bringing diverse stakeholders into a dialogue around a model can help in forming a shared understanding. Building a model can provide a discipline of thinking: the requirements of consistency and the need to use data can clarify how processes and systems really work.

Tako et al's study exemplifies the potential of simulation modelling to improve the allocation of scarce resources in the specific healthcare setting of an obesity service. More generally, however, this type of study illustrates the ways in which well constructed models can provide simplified representations of complex systems to yield practical insights into how a system will behave under different circumstances.

\section{Competing interests None.}

Provenance and peer review Not commissioned; externally peer reviewed.

\section{REFERENCES}

1 Tako AA, Kotiadis K, Vasilakis C, et al. Improving patient waiting times: a simulation study of an obesity care service. BMJ Qual Saf 2014;23:373-81.

2 Brailsford SC, Hilton NA. A comparison of discrete event simulation and system dynamics for modelling health care systems. In: Riley J. eds. Planning for the Future: Health Service Quality and Emergency Accessibility. Operational Research Applied to Health Services (ORAHS). Glasgow: Glasgow Caledonian University, 2001:18-39.
3 Taylor K, Lane D. Simulation applied to health services: opportunities for applying the system dynamics approach. J Health Serv Res Policy 1998;3:226-32.

4 Connelly LG, Bair AE. Discrete event simulation of emergency department activity: a platform for system-level operations research. Acad Emergency Med 2004;11:1177-84.

5 Davies R, Roderick P, Crabbe D, et al. A simulation to evaluate screening for Helicobacter pylori infection in the prevention of peptic ulcers and gastric cancers. Health Care Manag Sci 2002;4:249-58.

6 Atun RA, Lebcir RM, McKee M, et al. Impact of joined-up HIV harm reduction and multidrug resistant tuberculosis control programmes in Estonia: system dynamics simulation model. Health Policy 2007;81:207-17.

7 Bayer S, Barlow J, Curry R. Assessing the impact of a care innovation: telecare. Sys Dyn Rev 2007;23:61-80.

8 Ansah JP, Matchar DB, Love SR, et al. Simulating the impact of long-term care policy on family eldercare hours. Health Serv Res 2013;48:773-91.

9 Jahangirian M, Naseer A, Stergioulas L, et al. Simulation in health-care: lessons from other sectors. Oper Res Int J 2012;12:45-55.

10 Young T, Brailsford S, Connell C, et al. Using industrial processes to improve patient care. BMJ 2004;328:162-4.

11 Fone D, Hollinghurst S, Temple M, et al. Systematic review of the use and value of computer simulation modelling in population health and healthcare delivery. J Public Health Med 2003;25:325-35.

12 Brailsford SC, Vissers J. OR in healthcare: a European perspective. Eur J Oper Res 2011;2:223-34.

13 Brailsford SC, Harper PR, Patel B, et al. An analysis of the academic literature on simulation and modelling in healthcare. J Simulation 2009;3:130-40. 